\title{
Chess Expertise and the Fusiform Face Area: Why It Matters
}

\author{
Amy L. Boggan ${ }^{1}$ and Chih-Mao Huang ${ }^{2,3}$ \\ ${ }^{1}$ School of Behavioral and Brain Sciences, the University of Texas at Dallas, Dallas, Texas 75080, ${ }^{2}$ Department of Psychology, the University of Illinois at \\ Urbana-Champaign, Champaign, Illinois 61820, and ${ }^{3}$ Center for Vital Longevity, the University of Texas at Dallas, Dallas, Texas 75235 \\ Review of Bilalić et al.
}

Several brain regions have been linked to human face processing, most prominently the fusiform face area (FFA) in the fusiform gyrus. Dedicated architecture for face processing has also consistently been suggested by functional neuroimaging and single-unit recordings in primates. Faces are distinctive stimuli; they are a vital part of social interactions. Faces also are processed more holistically than other objects; face features and the relationships between them tend to be encoded all at once. Studies with infants and twins suggest a potential genetic component for specialized face processing. Such observations have led to the hypothesis that the FFA is an innate/dedicated face-processing area (i.e., the face-specificity hypothesis).

On the other hand, we are highly practiced at face recognition, as we must recognize associates among highly similar individuals. Also, experience appears to tune our ability to recognize different faces; people are generally better at recognizing faces of the race to which they were most exposed in childhood. Moreover, some studies have reported increased FFA activation when car experts and bird experts perceive cars and birds (Gauthier et al., 2000) and when radiologists examine scans (Harley et al., 2009). These observations have led to the hypothesis that the FFA is a holistic processor or individuator tuned by expertise (i.e., the expertise hypothesis).

Received Sept. 13, 2011; revised 0ct. 13, 2011; accepted 0ct. 17, 2011. We thank J. C. Bartlett for helpful comments on the manuscript.

Correspondence should be addressed to Amy Boggan, 800 West Campbell Road, GR 41, Richardson, TX 75083. E-mail: aboggan@utdallas.edu. DOI:10.1523/JNEUROSCI.4689-11.2011

Copyright $\odot 2011$ the authors $\quad 0270-6474 / 11 / 3116895-02 \$ 15.00 / 0$
Through extensive experience and deliberate practice, experts develop skills that transcend typical perception and memory limitations. Developing visual expertise shapes perception through top-down influences, and "chunking" mechanisms expand information capacity by grouping items into sets, reducing memory demands. Also, experts identify exemplars at more specific levels of categorization.

Clarifying the role of FFA is complicated by several difficulties that occur when attempting to compare face recognition with other-object recognition in experts: people are usually much more experienced with faces than other objects, other-object visual expertise is difficult to quantify, and other objects of expertise may share physical similarities and/or biological characteristics with faces. Studying chess experts, as was done in a recent article by Bilalić et al. in The Journal of Neuroscience, may overcome some of these problems.

Although chess configurations bear litthe featural or configural resemblance to faces, chess expertise, thought of as a cognitive skill, allows a critical test for the visual expertise hypothesis of FFA in several ways. First, while visually quite different from faces, chess evokes both part-based processing and rapid recognition of meaningful spatial relations between chess pieces among experts. Such part-based and more holistic processing are both critical for face recognition. Second, chess expertise is quantified statistically based on an international rating system, facilitating the assessment and verification of expertise.
Experience, particularly deliberate practice, is the strongest determinant of chess ratings (Charness et al., 2005). Finally, chess objects and their relative board positions lack biological characteristics and face-specific features, eliminating the possibility that FFA activation is related to face similarity. These factors make chess particularly well suited to investigate the influence of experience on expertise (Boggan et al., 2011).

Using functional magnetic resonance imaging, Bilalić et al. (2011) compared FFA activity in chess experts and novices during chess-relevant and chess-irrelevant tasks. Experiment 1 involved a recognition task with sequences of faces and naturalistic chess positions displayed either upright or inverted. Faces elicited greater FFA activation than chess displays in both experts and novices. Nonetheless, activity in the FFA was modulated by expertise, with chess experts demonstrating more activation than novices in response to chess stimuli. Surprisingly, however, both novices and chess experts exhibited as much or more FFA activation for inverted chess displays as for upright displays.

To clarify whether the FFA activity identified in Experiment 1 was related to sophisticated expertise-related recognition processes, Experiment 2 required participants to engage in three chess tasks, each with a different degree of relevance to chess skill. Chess structural relevance was varied as well, with normal (meaningful spatial relations among pieces) and random (pieces randomly distributed on the board) chess positions. Chess experts demonstrated greater FFA activation compared to 
novices in all conditions. Interestingly, FFA activation in experts was sensitive to structural relevance, particularly in the task most relevant to playing chess (Bilalić et al., 2011, their Fig. 2D). FFA modulation depended on the participant's expertise and the compatibility of the chess display with that expertise.

In Experiment 3, similar FFA effects were reported while controlling for visual complexity of chess stimuli. Consistent with Experiment 2, behavioral expertise effects were maximized in the most gamerelevant condition. Together, the results of Bilalić et al. (2011) demonstrated that FFA activity is modulated by expertise within a visual domain, the relevance of expertise to each task, and the typicality of viewed stimuli (i.e., whether the stimuli are well formed or abnormal). These findings provide a strong support for an expertise account of FFA function.

Because increased interest and motivation among experts regarding their area of expertise might be expected to increase attention in the chess-related tasks (McKone et al., 2007), right FFA activity in experts might reflect top-down neural processes, possibly confounding FFA expertise effects. To investigate whether topdown attentional processes influence any expertise effects in this study, Bilalić et al. (2011) monitored a specific control region of interest, the intraparietal sulcus (IPS), which has been linked to top-down attention. They reported that IPS activity was sensitive to task difficulty and structural relevance (normal or randomized positions), suggesting greater demands on top-down attentional control processes under these conditions. There were, however, no significant differences in IPS activation between chess experts and novices across all tasks and conditions, suggesting that FFA expertise effects reported by Bilalić et al. (2011) were independent of task difficulty and top-down modulation.

Bilalić et al. (2011) observed that FFA of chess experts was preferentially activated by ill formed (i.e., randomized) chess positions compared to well formed (i.e., normal) chess positions. This finding illustrates the need to better understand the precise role of the FFA. It is known that the FFA processes both holistic face representations and individual features (Liu et al., 2010). The perceptual field hypothesis for face recognition (Rossion, 2009) posits that holistic processing depends on access to information across broad regions of the face, and that such access is dependent on typically experienced face views (e.g., upright). When face presentations are atypical, humans are forced to rely on more part-based, featural strategies. To fully explore the role of the FFA in face recognition, it is important to establish to what extent FFA activation relates to the number and/or size of chess chunks, or typical constellations of chess pieces, among chess experts. Randomized game displays may preferentially activate the FFA through necessitating a greater number of smaller chunks, given that little or no contextual information is available across larger spans of the chessboard.

Another possible experience-related explanation for greater FFA response to randomized games displays is that the FFA is sensitive to stimulus familiarity. Recent studies have reported greater FFA activation when viewing unfamiliar faces than when viewing familiar faces (e.g., Minnebusch et al., 2009). Moreover, given that well formed chess positions contain meaningful configurations, it is also reasonable to posit that when chess experts engage in chess-specific task conditions, other brain regions, such as the precuneus (Krawczyk et al., 2011) and collateral sulci (Bilalić et al., 2010), reflect cognitive activity associated with chess chunks or templates.

In addition, extreme chess experts may differ qualitatively from less highly skilled players. Whereas Expert players (the accomplished players in Bilalić et al., 2011) are hypothesized to remember $\sim 1000$ chess chunks, Masters or higher (rated >2200) may remember as many as 100,000 (Simon and Gilmartin, 1973), which may allow them to observe meaningful configurations even within randomized games. Also, with increasing expertise, chunks are thought to consolidate into larger structures, templates, which means highly skilled players may perceive fewer, larger groupings of pieces within well formed games than less skilled players. A recent fMRI study using a similar task to Bilalić et al. (2011)'s Experiment 1 did not report a FFA expertise effect with chess international masters and grandmasters compared to novices (Krawczyk et al., 2011). Precise characterization of how expertise may tune face-sensitive regions is a critical challenge for future research.

In summary, Bilalić et al. (2011) showed that chess expertise modulates activity in the FFA. Rather than simply adding to previous findings with bird, car, Lepidoptera, and radiology experts, Bilalić et al. (2011) contrib- ute substantially to the expertise and face literatures in several ways. They examined strongly verified experts and manipulated both task relevance and whether positions were structurally relevant (typical) or ill formed (randomized). Bilalić et al. (2011)'s results clearly suggest that the FFA not only contributes to face recognition but also contributes to automatic holistic processing observed behaviorally among chess experts (Boggan et al., 2011). The advanced utilization of part-based and holistic processing by chess experts makes them an ideal group for further study of complex visual processes. As investigations of FFA function proceed from different approaches, determining how expertise qualitatively and quantitatively shapes the function of FFA remains a key challenge.

\section{References}

Bilalić M, Langner R, Erb M, Grodd W (2010) Mechanisms and neural basis of object and pattern recognition: a study with chess experts. J Exp Psychol Gen 139:728-742.

Bilalić M, Langner R, Ulrich R, Grodd W (2011) Many faces of expertise: fusiform face area in chess experts and novices. J Neurosci 31:10206-10214.

Boggan AL, Bartlett JC, Krawczyk DC (2011) Chess masters show a hallmark of face processing with chess. J Exp Psychol Gen. Advance online publication. doi:10.1037/a0024236.

Charness N, Tuffiash M, Krampe R, Reingold E, Vasyukova E (2005) The role of deliberate practice in chess expertise. Appl Cogn Psychol 19:151-165.

Gauthier I, Skudlarski P, Gore JC, Anderson AW (2000) Expertise for cars and birds recruits brain areas involved in face recognition. Nat Neurosci 3:191-197.

Harley EM, Pope WB, Villablanca JP, Mumford J, Suh R, Mazziotta JC, Enzmann D, Engel SA (2009) Engagement of fusiform cortex and disengagement of lateral occipital cortex in the acquisition of radiological expertise. Cereb Cortex 19:2746-2754.

Krawczyk DC, Boggan AL, McClelland MM, Bartlett JC (2011) Brain organization of perception in chess experts. Neurosci Lett 499:64-69.

Liu J, Harris A, Kanwisher N (2010) Perception of face parts and face configurations: an fMRI study. J Cogn Neurosci 22:203-211.

McKone E, Kanwisher N, Duchaine BC (2007) Can generic expertise explain special processing for faces? Trends Cogn Sci 11:8-15.

Minnebusch DA, Suchan B, Köster O, Daum I (2009) A bilateral occipitotemporal network mediates face perception. Behav Brain Res 198:179-185.

Rossion B (2009) Distinguishing the cause and consequence of face inversion: The perceptual field hypothesis. Acta Psychologica 132:300-312.

Simon HA, Gilmartin K (1973) A simulation of memory for chess positions. Cogn Psychol 5:29-46. 